\title{
Correlation between fine needle aspiration and intraoperative frozen section findings for thyroid patients; a single center study
}

\author{
Azar Baradaran $^{\mathbb{D}}$, Maryam Derakhshan ${ }^{\mathbb{D}}$, Negin Ghanbari* ${ }^{*}$ \\ Department of Pathology, School of Medicine, Isfahan University of Medical Sciences, Isfahan, Iran
}

Correspondence to:

Negin Ghanbari, Email: ghanbari.

negin21@gmail.com

Received: 1 Aug. 2021

Accepted: 8 Oct. 202

ePublished: 25 Oct. 2021

Keywords: Fine needle aspiration, Intraoperative frozen section, Thyroid

\begin{abstract}
Introduction: Thyroid nodules are one of the most common diseases.

Objectives: The aim of this study was to evaluate the correlation between fine needle aspiration (FNA) and intraoperative frozen section findings for thyroid disease patients.

Patients and Methods: In this study, the FNA and intraoperative frozen section findings of 143 patients who were admitted to an educational hospital, Isfahan, Iran were collected and classified using these diagnostic methods during 2015. To find the correlation between FNA and intraoperative frozen section findings, statistical analysis was conducted using SPSS software version 16.0 (Chicago, IL).

Results: Sensitivity and specificity of the positive FNA findings were $71 \%$ and $86.8 \%$, respectively $(P<0.001)$. The maximum concordance between FNA and intraoperative frozen section for papillary thyroid cancer was seen $(P<0.001)$.

Conclusion: In this study, a significant association between FNA and intraoperative frozen section of thyroid cancers, among our patients, however this finding requires further investigation by larger studies.
\end{abstract}

\begin{abstract}
Citation: Baradaran A, Derakhshan M, Ghanbari N. Correlation between fine needle aspiration and intraoperative frozen section findings for thyroid patients; a single center study. J Prev Epidemiol. 2022;7(1):e04. doi: $10.34172 /$ jpe. 2022.04
\end{abstract}

\section{Introduction}

Thyroid nodules are one of the most common diseases. In Iran, 9.5\% of the adult population has a palpable thyroid nodule (1). This figure is equivalent to 1 to 2 cases per 400000 population, which is $1 \%$ of cancers and $0.5 \%$ of cancer deaths (2).

Thyroid nodules are solid or fluid-filled masses that form inside the thyroid and form a small gland at the base of the neck. Many of these nodules are asymptomatic (1); however some thyroid nodules may become so large that they put pressure on the trachea, causing breathing problems and difficulty swallowing (2).

Methods for diagnosing thyroid nodules, in addition to accurate touch by a physician, include ultrasound, isotope scanning, fine needle aspiration (FNA) and more recently, Doppler ultrasound, and also examination of intraoperative frozen thyroid surgery specimens $(3,4)$. FNA is widely used to diagnose thyroid malignancies (4). However, there is still no consensus on the diagnostic value of these samples, applications, sensitivity, specificity and accuracy of diagnosis in thyroid nodular lesions (4). As reported in some previous studies, only in suspected cases such as

\section{Key point}

In our study, the FNA and intraoperative frozen section findings of thyroid glands of 143 thyroid cancer patients were assessed. We found a significant association between FNA and intraoperative frozen section of thyroid cancers.

papillary, undifferentiated, and medullary cancers, FNA samples can be effective in confirming the lesion and determining the surgical technique (5). However, in the case of follicular lesions, Hurthle cell thyroid cancer and tuberculosis, thyroid FNA specimens do not help much and require a pathological evaluation for a more detailed examination (5). In this regard, in a study conducted on 42 patients with thyroid nodules, the results showed that the FNA method in the diagnosis of thyroid nodules is a more sensitive and specific method than the surgical sampling method (6). According to the results of this study, FNA has played a very important role in reducing additional and unnecessary surgeries (6). In another study by Iannuccilli et al, on thyroid nodules in terms of size, internal vascularity, echogenicity, and calcification, the need for FNA on all nodules with internal

Copyright (C) 2022 The Author(s); Published by Society of Diabetic Nephropathy Prevention. This is an open-access article distributed under the terms of the Creative Commons Attribution License (http://creativecommons.org/licenses/by/4.0), which permits unrestricted use, distribution, and reproduction in any medium, provided the original work is properly cited. 
calcification was demonstrated $(7,8)$. At present, due to the uncertainty of the exact match between FNA findings and thyroid surgery, samples are taken from patients with suspected malignancy FNA findings or even from a group who have to undergo surgery despite benign FNA report becomes. This increases the cost and risks associated with performing a biopsy. Therefore, conducting a study to find consistency between FNA findings and thyroid surgery can provide valuable information to medical professionals. This valuable information greatly facilitates treatment optimization.

\section{Objectives}

As far as the researchers of this study know, so far no comprehensive demographic study has been conducted on the compatibility of FNA findings and thyroid surgery in patients in Isfahan province. Therefore, a study in this regard can lead to a suitable and practical solution in improving the quality of diagnostic and therapeutic services. The aim of this study was to evaluate the correlation between the findings of FNA and thyroid surgery samples in patients of our hospital in Isfahan from 2015 to 2016.

\section{Patients and Methods \\ Study design}

This study was an observational, descriptive-analytical, cross-sectional and directionless study that was conducted in 2015 in the pathology department of Al-Zahra hospital in Isfahan. The statistical population of the study was the FNA reports in the archives of the above section.

According to statistical calculations and similar previous research, in this study, a sample of 143 patients was examined.

By referring the researcher to the information unit related to the file and electronic archive of the pathology department of this hospital, the FNA reports of the patients were extracted and classified based on specific diagnoses (benign or malignant). These reports were related to patients who had malignant FNA or had to undergo thyroidectomy. Surgical sampling was performed for these patients. Surgical specimens were also evaluated for patients with benign FNA who required surgery due to cosmetic and respiratory problems. Finally, all findings from surgical samples of thyroid tissue were compared with the findings from the relevant FNA. The studied variables including patients' age and gender were extracted from patients' files and collected in special forms.

\section{Data analysis}

The obtained data were finally entered into the computer and analyzed by SPSS software (version 20, Chicago, IL, USA) and chi-square test. $P$ value $<0.05$ was considered significant.

\section{Results}

Among 143 patients study patients, data related to 121 were $(84.6 \%)$ males. The mean age of patients was 44.9 years (18-78 years). We found that positive FNA findings for papillary carcinoma of the thyroid, nodular goiter, cyst and Hashimoto's thyroiditis were 49 (38\%), 39 (30.2\%), 7 (5.4\%) and $4(3.1 \%)$, respectively. Regarding surgery, the positive results for the mentioned diseases were 76 (53\%), $40(28 \%), 1(0.7 \%)$ and $3(2.1 \%)$ cases, respectively.

In general, the sensitivity and diagnostic specificity of FNA in detecting abnormalities of the thyroid, compared with the samples obtained from surgery, were $71 \%$ and $86.8 \%$, respectively, which is statistically significant $(P<0.001$; Table 1$)$.

In addition, the correlation of each of the FNA findings including papillary carcinoma of the thyroid gland, nodular goiter, cysts and Hashimoto's thyroiditis, compared to its surgical findings was $34.1 \%, 18.6 \%, 0.8 \%$ and $1.6 \%$, respectively. Our study showed that the highest correlation between FNA and surgery findings was in papillary thyroid carcinoma $(P<0.001)$.

Tables 2 and 3 show the findings of FNA and surgery for benign, malignant, and unknown cases, respectively.

Table 4 shows the results of the association between FNA and surgery for papillary carcinoma of the thyroid, nodular goiter, cyst, and Hashimoto's thyroiditis.

\section{Discussion}

Recently, the use of ultrasound devices to perform guided FNA is very common $(9,10)$. Therefore, comparing the results of FNA under ultrasound guidance and thyroid surgery samples to examine its nodules is of particular importance and probably has a significant effect on how to choose the appropriate treatment (11).

In this study, the correlation between the findings of biopsy and thyroid surgery samples in patients of an educational hospital in Isfahan in 2015 was investigated. A significant correlation between the findings of FNA and the histology of the related diseases was observed, while in other studies, FNA has shown considerable weakness in diagnosing malignant or benign thyroid nodules, indicating the necessity for FNA $(3,12-14)$. The results

Table 1. FNA diagnostic compliance with its surgical specimens

\begin{tabular}{|c|c|c|c|c|c|c|c|c|}
\hline \multirow{2}{*}{ Finding FNA } & & \multicolumn{2}{|c|}{ Finding histopathology } & \multirow{2}{*}{ Sensitivity } & \multirow{2}{*}{ Specificity } & \multirow{2}{*}{ PPV } & \multirow{2}{*}{ NPV } & \multirow{2}{*}{$P$ value } \\
\hline & & Benign & Malignant & & & & & \\
\hline \multirow{2}{*}{ Papillary thyroid carcinoma } & Benign & 57 (51.4\%) & $54(48.6 \%)$ & \multirow{2}{*}{$71 \%$} & \multirow{2}{*}{$86.8 \%$} & \multirow{2}{*}{$89.8 \%$} & \multirow{2}{*}{$64.7 \%$} & \multirow{2}{*}{$<0.001$} \\
\hline & Malignant & $23(26.7 \%)$ & $63(73.3 \%)$ & & & & & \\
\hline
\end{tabular}

PPV, positive predictive value; NPV, negative predictive value. 
Table 2. Results of FNA study

\begin{tabular}{|c|c|c|c|c|c|}
\hline & & Frequency & Percent & Valid Percent & Cumulative Percent \\
\hline \multirow{4}{*}{ Valid } & Benign & 52 & 36.4 & 36.4 & 36.4 \\
\hline & Malignant & 47 & 32.9 & 32.9 & 69.2 \\
\hline & Undetermined & 44 & 30.8 & 30.8 & 100.0 \\
\hline & Total & 143 & 100.0 & 100.0 & \\
\hline
\end{tabular}

Table 3. Results of surgical examination

\begin{tabular}{|c|c|c|c|c|c|}
\hline & & Frequency & Percent & Valid Percent & Cumulative Percent \\
\hline \multirow{4}{*}{ Valid } & Benign & 59 & 41.3 & 41.3 & 41.3 \\
\hline & Malignant & 82 & 57.3 & 57.3 & 98.6 \\
\hline & Undetermined & 2 & 1.4 & 1.4 & 100.0 \\
\hline & Total & 143 & 100.0 & 100.0 & \\
\hline
\end{tabular}

Table 4. Results of the agreement between FNA and surgery for papillary thyroid carcinoma, nodular goiter, cyst and Hashimoto's thyroiditis

\begin{tabular}{|c|c|c|c|c|}
\hline & Sensitivity & Specificity & PPV & NPV \\
\hline Papillary thyroid carcinoma & $57.9 \%$ & $92.5 \%$ & $89.8 \%$ & $66 \%$ \\
\hline Goiter nodular & $60 \%$ & $85.4 \%$ & $61.5 \%$ & $84.6 \%$ \\
\hline Cyst & $100 \%$ & $95.8 \%$ & $14.3 \%$ & $100 \%$ \\
\hline Hashimoto's thyroiditis & $66.7 \%$ & $98.6 \%$ & $50 \%$ & $99.3 \%$ \\
\hline
\end{tabular}

of our study showed that in general, the sensitivity and diagnostic specificity of FNA in detecting abnormalities of the thyroid, compared with the samples obtained from surgery were $71 \%$ and $86.8 \%$, respectively. In the case of benign or malignant segregation of thyroid nodules, our results showed that FNA reports correlated with surgical outcomes and had a significant degree of agreement (Tables 2 and 3).

In suspected cases of FNA in papillary, undifferentiated and medullary cancers, performing thyroid surgery specimens can be effective in confirming the lesion and determining the surgical plan (5), since, in cases of follicular lesions and Hurthle cell carcinoma, thyroid FNA samples do not help much and we have to wait for a definitive pathology response (5).

\section{Conclusion}

According to our findings, the highest correlation between FNA and surgery findings was in the case of papillary thyroid carcinoma. However, larger multi-centric studies are required to confirm our results.

\section{Limitations of the study}

Our study has a single-center nature which requires to be considered by larger population.

\section{Authors' contribution}

$A B, M D$ and $N G$ were the principal investigators of the study. $A B$, $M D$ and $N G$ were included in preparing the concept and design. MAS and SA revisited the manuscript and critically evaluated the intellectual contents. All authors participated in preparing the final draft of the manuscript, revised the manuscript and critically evaluated the intellectual contents. All authors have read and approved the content of the manuscript and confirmed the accuracy or integrity of any part of the work.

\section{Ethical issues}

The research followed the tenets of the Declaration of Helsinki. The ethics committee of Isfahan university of medical sciences approved this study. The institutional ethical committee at Isfahan University of Medical Sciences approved all study protocols (\#IR. MUI.REC.1396.058). Accordingly, written informed consent was taken from all participants before any intervention. This study was extracted from M.D., thesis of Negin Ghanbari at this university (Thesis \#396058). Besides, ethical issues (including plagiarism, data fabrication, double publication) have been completely observed by the authors.

Conflicts of interest

The authors declare that they have no competing interests.

Funding/Support

None.

References

1. Azizi F. Guide for diagnosis and treatment of thyroid nodules. Guidelines collection for diagnosis and treatment of endocrine glands disease and metabolism. 1st ed. 2004. p. 4-21.

2. Hegedüs L. Clinical practice. The thyroid nodule. N Engl J Med. 2004;351:1764-71. doi: 10.1056/NEJMcp031436.

3. Mortensen JD, Woolner LB, Bennett WA. Gross and microscopic findings in clinically normal thyroid glands. J Clin Endocrinol Metab. 1955;15:1270-80. doi: 10.1210/jcem-1510-1270.

4. Ogilvie JB, Piatigorsky EJ, Clark OH. Current status of fine needle aspiration for thyroid nodules. Adv Surg. 2006;40:22338. doi: 10.1016/j.yasu.2006.06.003.

5. Chang HY, Lin JD, Chen JF, Huang BY, Hsueh C, Jeng LB, et al. Correlation of fine needle aspiration cytology and frozen section biopsies in the diagnosis of thyroid nodules. J Clin Pathol. 1997;50:1005-9. doi: 10.1136/jcp.50.12.1005.

6. Mittendorf EA, Tamarkin SW, McHenry CR. The results of ultrasound-guided fine-needle aspiration biopsy for evaluation 
of nodular thyroid disease. Surgery. 2002;132:648-53. doi: 10.1067/msy.2002.127549.

7. Iannuccilli JD, Cronan JJ, Monchik JM. Risk for malignancy of thyroid nodules as assessed by sonographic criteria: the need for biopsy. J Ultrasound Med. 2004;23:1455-64. doi: 10.7863/ jum.2004.23.11.1455.

8. Haugen BR, Alexander EK, Bible KC, Doherty GM, Mandel SJ, Nikiforov YE, et al. 2015 American Thyroid Association Management Guidelines for Adult Patients with Thyroid Nodules and Differentiated Thyroid Cancer: The American Thyroid Association Guidelines Task Force on Thyroid Nodules and Differentiated Thyroid Cancer. Thyroid. 2016;26:1-133. doi: 10.1089/thy.2015.0020.

9. Cibas ES, Ali SZ; NCl Thyroid FNA State of the Science Conference. The Bethesda System For Reporting Thyroid Cytopathology. Am J Clin Pathol. 2009;132:658-65. doi: 10.1309/AJCPPHLWMI3JV4LA.

10. Mufti ST, Molah R. The bethesda system for reporting thyroid cytopathology: a five-year retrospective review of one center experience. Int J Health Sci (Qassim). 2012;6:159-73. doi: 10.12816/0005991.

11. Russ G, Bigorgne C, Royer B, Rouxel A, Bienvenu-Perrard M. [The Thyroid Imaging Reporting and Data System (TIRADS) for ultrasound of the thyroid]. J Radiol. 2011;92:701-13. doi: 10.1016/j.jradio.2011.03.022.

12. Farshchian N, M.F., Izadi B, Rahimi M., The concordance between thyroid nodules ultrasound, based on thyroid Imaging reporting and data systems, and fine needle aspiration. Journal of Kermanshah University of Medical Sciences. 2016;19:e69755. doi: 10.22110/jkums.v19i7.2524.

13. Bomeli SR, LeBeau SO, Ferris RL. Evaluation of a thyroid nodule. Otolaryngol Clin North Am. 2010;43:229-38. doi: 10.1016/j.otc.2010.01.002.

14. Hosseini M, Alizadeh Otaghvar HR, Tizmaghz A, Shabestanipour G, Arvaneh S. Evaluating the accuracy of fine needle aspiration and frozen section based on permanent histology in patients with follicular lesions. Med J Islam Repub Iran. 2015;29:239. 